\title{
Knockdown of biglycan expression by RNA interference inhibits the proliferation and invasion of, and induces apoptosis in, the HCT116 colon cancer cell line
}

\author{
XIAOJING XING ${ }^{1,2}$, XIAOHU $\mathrm{GU}^{3}$ and TIANFEI MA ${ }^{1}$ \\ ${ }^{1}$ Cancer Prevention and Treatment Office; ${ }^{2}$ Department of Internal Oncology; ${ }^{3}$ Department of Surgical Oncology, \\ Liaoning Cancer Hospital and Institute, Shenyang, Liaoning 110042, P.R. China
}

Received December 2, 2014; Accepted August 24, 2015

DOI: $10.3892 / \mathrm{mmr} .2015 .4383$

\begin{abstract}
Biglycan is an important component of the extracellular matrix, and it is also a member of small leucine-rich proteoglycan family. Previous studies indicated that the expression of biglycan was increased in a variety of tumor tissues, including colon cancer. However, the mechanisms underlying its effects in colon cancer remain to be fully elucidated. In the present study, the effects of biglycan knockdown on colon cancer cell proliferation, migration, invasion and apoptosis were investigated. The mRNA expression levels of biglycan in the HCT116 colon cancer cell line were downregulated using RNA interference, and the stably transfected cell line was obtained through G418 screening for subsequent experiments. The results revealed that downregulation of the expression of biglycan suppressed cell proliferation and caused a cell cycle arrest at the G0/G1 phase. The results of the western blot analysis also revealed that the expression levels of cell cycle-associated proteins, including cyclin A and cyclin D1, were markedly decreased following silencing of biglycan, whereas the expression levels of p21 and p27 were markedly increased compared with that of the short hairpin RNA control group. Furthermore, the decreased expression of biglycan inhibited colon cancer cell migration and invasion, and induced apoptosis. A complete inhibition of the p38 signaling pathway with SB203580 effectively reversed the increase in apoptotic cell numbers induced by biglycan downregulation. Taken together, the results of the present study indicated that biglycan exerts an important role in cell proliferation, migration, invasion and apoptosis in colon cancer, and that biglycan regulates the p38 MAPK signaling pathway by exerting an antiapoptotic effect. Therefore, biglycan may represent a putative target for colon cancer gene therapy.
\end{abstract}

Correspondence to: Dr Xiaojing Xing, Cancer Prevention and Treatment Office, Liaoning Cancer Hospital and Institute, 44 Xiaoheyan Road, Shenyang, Liaoning 110042, P.R. China E-mail:1nszl_xxj@163.com

Key words: biglycan, colon cancer cell, invasion, proliferation, apoptosis

\section{Introduction}

Colon cancer is one of the most common types of gastrointestinal cancer, endangering human health (1). It was previously reported that $\sim 650,000$ individuals worldwide suffer mortality from colon cancer annually, and the number of diagnoses and mortality continue to increase annually $(2,3)$. Despite the continual emergence of early screening methods and novel chemotherapeutic strategies, the survival rate of patients with colon cancer has failed to markedly improved over the last 20 years (4). Therefore, an in-depth investigation into the mechanisms underlying the pathogenesis of colon cancer is crucial for the identification of effective treatment strategies, and improvements in the survival rate of patients.

Biglycan is an important member of the small leucine-rich proteoglycan family, which is expressed on the cell surface and in the extracellular matrix (5). Previous studies indicated that a lack of biglycan was associated with reduced bone mass and osteoporosis $(6,7)$. Previous reports also demonstrated that the expression of biglycan was increased in multiple types of tumor tissue, including liver (8), ovarian (9), endometrial (10), pancreatic (11), gastric (12) and colon cancer (13). These findings suggest an important role for biglycan in the development of cancer, however, the underlying mechanism in colon cancer remains to be fully elucidated.

The present study aimed to investigate the expression of biglycan in HCT116 colon cancer cells using biglycan-specific short hairpin (sh)RNA. A stably transfected cell line was established through G418 screening, and the effects of biglycan on colon cancer cell proliferation, migration, invasion and apoptosis were investigated. Furthermore, how biglycan may be a potential target for colon cancer gene therapy was discussed.

\section{Materials and methods}

Cell culture.Human HCT116 colon cancer cells were purchased from the Shanghai Institute of Cell Biology, Chinese Academy of Sciences (Shanghai, China). The cells were cultured in McCoy's 5A medium (Sigma-Aldrich, St. Louis, MO, USA), supplemented with $10 \%$ fetal bovine serum (FBS; GE Healthcare Life Sciences HyClone Laboratories, Logan, UT, USA) at $37^{\circ} \mathrm{C}$ with $5 \% \mathrm{CO}_{2}$. The culture medium was changed 
every 2-3 days, and the cells were digested with $0.25 \%$ trypsin (Beyotime Institute of Biotechnology, Haimen, China) for passage when $80 \%$ confluence was reached.

Plasmid construction. The biglycan-specific shRNA plasmid was constructed using the pGCsi-H1 plasmid (Shanghai GeneChem Co. Ltd., Shanghai, China). The human biglycan mRNA sequence (NM_001711.4) was obtained from the National Center for Biotechnology Information database (http:/www.ncbi.nlm.nih.gov/nuccore/NM_001711.4). A total of four biglycan shRNA interference sequences, in addition to a sequence of an identical length, which was not associated with the human genome sequences (serving as the negative control), were generated, according to the principles of shRNA design: shRNA-biglycan-1, 5'-GATCCCCGATCTCCAAGA TCCATGAGTTCAAGAGACTCATGGATCTTGGAGATC TTTTT-3' (forward); shRNA-biglycan-2, 5'-GATCCCCGC TCTACATCTCCAAGAACTTCAAGAGAGTTCTTGGAG ATGTAGAGCTTTTT-3' (forward); shRNA-biglycan-3, 5'-GATCCCCGCTCAACTACCTGCGCATCTTCAAGAGAG ATGCGCAGGTAGTTGAGCTTTTT-3' (forward); and shRNA-biglycan-4, 5'-GATCCCCATCCAGGCCATCGA ACTGGTTCAAGAGACCAGTTCGATGGCCTGGATTTT TT-3' (forward). The shRNA fragments were subsequently ligated into the pGCsi-H1 vector between the HindIII and BamHI sites. The constructs obtained were verified through double restriction enzyme digestion (Fermentas, Vilnius, Lithuania) and sequencing, and these were termed shRNA-biglycan 1, 2, 3 and 4, or shRNA-control.

Cell transfection and stable cell line screening. The HCT116 cells were seeded into six-well plates at a density of $1.0 \times 10^{5}$ cells/well, and were cultured overnight until $70-80 \%$ confluence was reached. The shRNA-biglycan 1, 2, 3 and 4 co nstructs or the shRNA control plasmid were subsequently transfected into the cells using Lipofectamine ${ }^{\mathrm{TM}} 2000$ (Invitrogen Life Technologies, Carlsbad, CA, USA), according to the manufacturer's instructions. G418 (Invitrogen Life Technologies) was added to the culture media at $24 \mathrm{~h}$ following transfection to screen for stably transfected cells. G418-resistant individual clones were retrieved at 4 weeks post-treatment. Following confirmation of the expression of biglycan, the selected stable clones were continuously cultured in G418-containing medium, and expanded for subsequent experiments.

Reverse transcription-quantitative polymerase chain reaction $(R T-q P C R)$. The total RNA was extracted from the cells using TRIzol ${ }^{\circledR}$ reagent (Invitrogen Life Technologies). The RNA was reverse-transcribed into cDNA using the first-strand cDNA synthesis kit (Takara Bio, Inc., Dalian, China), according to the manufacturer's instructions. The primer sequences were as follows: Biglycan, upstream: 5'-GGTCTCCAGCACCTCTAC GCC-3' and downstream: 5'-AACACTCCCTTGGGCACC TT-3'; $\beta$-actin, upstream: 5'-CTTAGTTGCGTTACACCCTTT CTTG-3' and downstream: 5'-CTGTCACCTTCACCGTTC CAGTTT-3'. The RT-qPCR reactions were performed using an Exicycler ${ }^{\mathrm{TM}} 96$ quantitative fluorescence analyzer (Bioneer Corporation, Daejeon, Korea). The PCR reactions consisted of an initial step at $95^{\circ} \mathrm{C}$ for $10 \mathrm{~min} ; 40$ cycles of $95^{\circ} \mathrm{C}$ for $10 \mathrm{sec}, 58^{\circ} \mathrm{C}$ for $20 \mathrm{sec}$ and $72^{\circ} \mathrm{C}$ for $30 \mathrm{sec}$; and a final step at $4^{\circ} \mathrm{C}$ for $5 \mathrm{~min}$.
Western blotting. The cells were lysed using radioimmunoprecipitation lysis buffer (Beyotime Institute of Biotechnology), and the protein concentration of each sample was measured using the bicinchoninic acid method. Equal quantities of protein $(40 \mu \mathrm{g})$ were separated by $13 \%$ SDS-PAGE (Solarbio Science and Technology Co.,Ltd, Beijing, China) for caspase-3, p21 and p27, or by $10 \%$ SDS-PAGE in all other cases and subsequently transferred onto polyvinylidene difluoride membranes (EMD Millipore, Bedford, MA, USA). The membrane was subsequently blocked with 5\% non-fat dry milk at room temperature for $1 \mathrm{~h}$, followed by incubation with the following diluted primary antibodies: Rabbit polyclonal anti-biglycan antibody (cat no. sc-33788; 1:100; Santa Cruz Biotechnology, Inc., Dallas, TX, USA), rabbit polyclonal anti-caspase-3 antibody (cat no. w101992a; 1:1,000), rabbit polyclonal anti-p27 antibody (cat no. wl01769; 1:1,000), rabbit polyclonal anti-cyclin A antibody (cat no. w101753; 1:200), rabbit polyclonal anti-p21 antibody (cat no. wl0362; 1:100), rabbit polyclonal anti-cyclin D1 antibody (cat no. wl01435a; 1:100) (all from Wanleibio, Shenyang, China), rabbit polyclonal anti-p38 antibody (cat no. sc-7149; 1:100) and anti-phosphorylated-p38 antibody (cat no. sc-101758; 1:100) (both from Abcam, Cambridge, MA, USA) at $4{ }^{\circ} \mathrm{C}$ overnight. The membrane was subsequently incubated with horseradish peroxidase (HRP)-conjugated goat anti-rabbit immunoglobulin G (cat no. A0208; 1:5,000; Beyotime Institute of Biotechnology) at $37^{\circ} \mathrm{C}$ for $1 \mathrm{~h}$. $\beta$-actin was used as an internal loading control, for which HRP-conjugated monoclonal mouse anti- $\beta$-actin antibody (cat no. KC-5A08; 1:10,000; Kangchen Biotech, Shanghai, China) was used. An enhanced chemiluminescence substrate (7 Sea Pharmtech, Shanghai, China) was added to the membrane in the dark for image development, and the scanned images were subsequently analyzed using Image $\mathrm{J}$ software (version 1.35; National Institutes of Health, Bethesda, MD, USA).

Cell counting kit-8 (CCK-8) assay. The cells were counted and seeded into 96 -well plates $\left(2 \times 10^{4}\right.$ cells/well) and subsequently cultured as before. Aliquots of $10 \mu \mathrm{l} \mathrm{CCK}-8$ solution (Beyotime Institute of Biotechnology) were added to the cell cultures at different time points, and incubated at $37^{\circ} \mathrm{C}$ for $1 \mathrm{~h}$. The absorbance at $450 \mathrm{~nm}$ was measured for each well using a microplate reader (ELx800; Bio-Tek Instruments, Inc., Winooski, VT, USA).

Scratch wound assay. The cells of each group were seeded into 6 -well plates $\left(1 \times 10^{5}\right.$ cells/well). The cells, which had reached $80 \%$ confluence, were used for the assay. The culture medium was discarded and a $200 \mu$ l pipette tip was used to perpendicularly scratch a wound on the surface of the cell monolayer. The cells were subsequently washed twice with serum-free culture media and cultured in McCoy's 5A medium, containing $10 \%$ FBS. Images of the cells were captured at 0,12 and $24 \mathrm{~h}$ following the scratching procedure using a phase-contrast microscope (Motic AE31; Motic China, Xiamen, China), in order to calculate the distances that the cells had migrated into the denuded area.

Transwell assay. Transwell chambers (Corning Life Sciences, Tewksbury, MA, USA) coated with Matrigel (BD Biosciences, Franklin Lakes, NJ, USA) were used for the cell invasion assay. 
A total of $5 \times 10^{4}$ cells were collected from each group and resuspended in $200 \mu \mathrm{l}$ serum-free cell culture medium. The cells were subsequently seeded into the upper Transwell chamber, and $800 \mu \mathrm{l}$ McCoy's 5A culture medium, containing 10\% FBS, was added to the lower chamber. Following a $24 \mathrm{~h}$ incubation, the cells in the upper chamber were removed using a cotton bud, and the cells present on the lower surface of the insert were fixed and stained with $0.5 \%$ crystal violet dye (Amresco, Inc., Solon, OH, USA). Subsequently, the cells were washed, images were captured and the number of cells was quantified under an inverted microscope (Motic AE31; Motic China).

Flow cytometric analysis. The effect of biglycan on the cell cycle progression and cell apoptosis associated with colon cancer was measured by flow cytometry. Briefly, the cells were trypsinized and fixed with $70 \%$ pre-cooled ethanol for $2 \mathrm{~h}$, prior to the re-suspension of the collected cells using staining solution containing $50 \mathrm{mg} / \mathrm{ml}$ propidium iodide (PI) and $1 \mathrm{mg} / \mathrm{ml}$ RNAse A (Beyotime Institute of Biotechnology) in $1 \mathrm{ml}$ sodium citrate buffer. The cells were subsequently analyzed for cell cycle distribution using a BD FACSCalibur flow cytometer (BD Biosciences). Cellular apoptosis was detected using the annexin V-fluorescein isothiocyanate (FITC)/PI apoptosis detection kit (Nanjing KeyGen Biotech., Co., Ltd., Nanjing, China), according to the manufacturer's instructions. Cells which had reached $80 \%$ confluence were treated with trypsin and collected. Following washing with phosphate-buffered saline, the cells were resuspended in $500 \mu 1$ annexin V-binding buffer (Nanjing KeyGen Biotech). Subsequently, $5 \mu$ l FITC-labeled annexin V and $5 \mu \mathrm{l}$ PI dye were added the cells, prior to an incubation at room temperature in the dark for $15 \mathrm{~min}$. The cells were analyzed by flow cytometry to determine the degree of cellular apoptosis.

Statistical analysis. All experimental data are expressed as the mean \pm standard deviation. One-way analysis of variance was used for paired comparisons between groups, and the Bonferroni post-hoc test was used for multiple comparisons. Graphpad Prism 5.0 software (GraphPad Software, Inc., La Jolla, CA, USA) was used for the data analysis and for generating the graphs. $\mathrm{P}<0.05$ was considered to indicate a statistically significant difference.

\section{Results}

Construction of a stable HCT116 cell line with biglycan silencing. To assess the role of biglycan in colon cancer cells, biglycan-specific shRNA vectors were initially constructed, which were subsequently transfected into HCT116 colon cancer cells. The mRNA expression levels of biglycan in each group of cells was determined by RT-qPCR and western blot analysis. As shown in Fig. 1, all the biglycan-specific shRNA fragments downregulated the mRNA expression level of biglycan in the transfected cells, compared with the shRNA-control group $(\mathrm{P}<0.05$ or $\mathrm{P}<0.01)$, among which shRNA-biglycan 4 exhibited the greatest efficiency of interference (the percentage efficiency values for the downregulation were $71.33 \pm 1.53 \%$ for biglycan mRNA, and $72.33 \pm 3.51 \%$ for biglycan protein levels). On the basis of these results, the cells which were stably transfected with shRNA-biglycan 4 were selected for subsequent experiments.

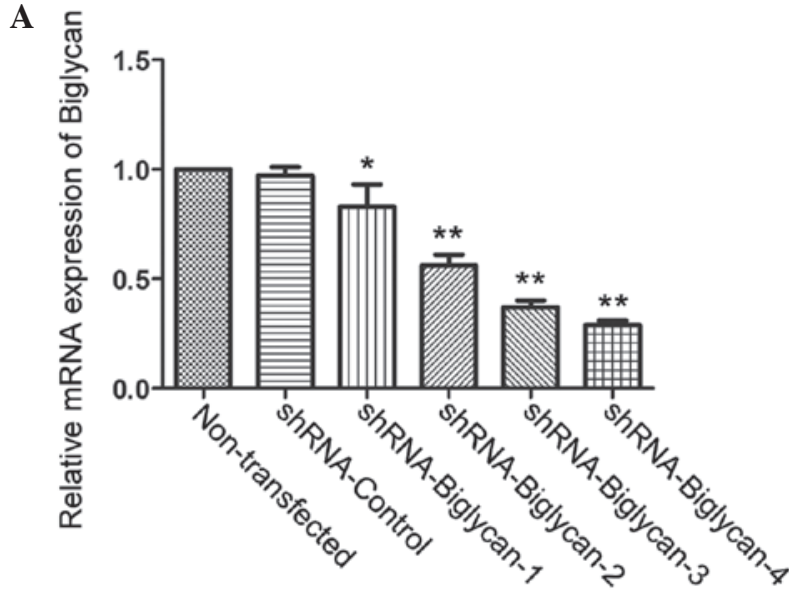

B

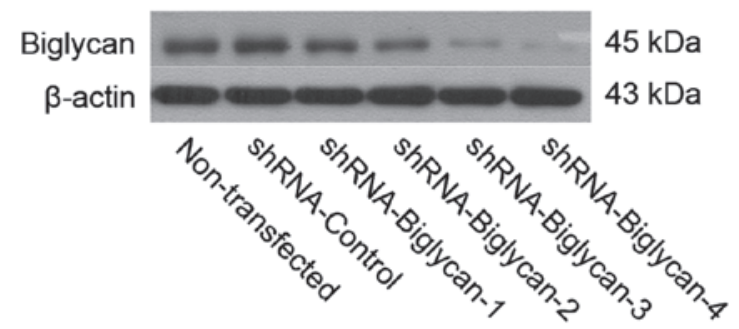

C

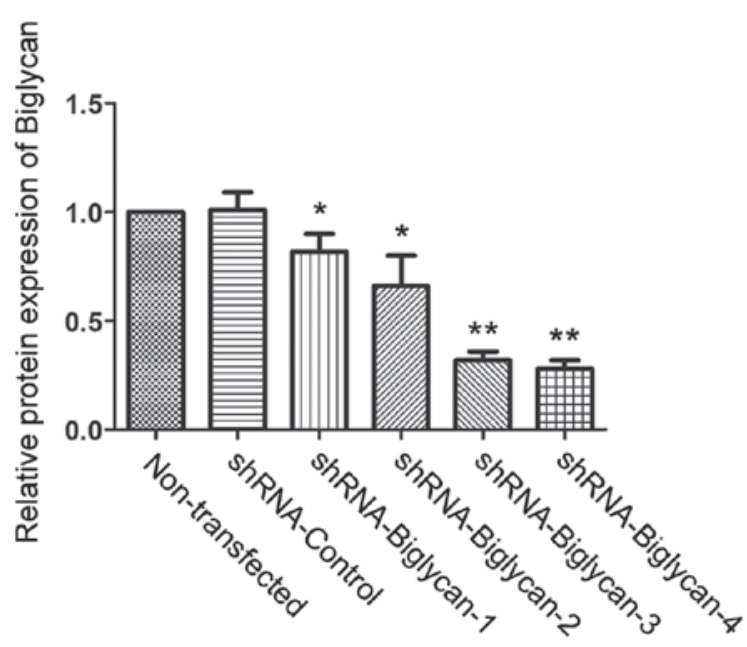

Figure 1. Construction of the stable HCT116 cell line with biglycan downregulation. The (A) mRNA and (B) protein expression levels of biglycan in the shRNA-biglycan/control-transfected or non-transfected cells was detected using reverse transcription-quantitative polymerase chain reaction and western blotting, respectively. (C) The relative protein expression of biglycan was normalized against $\beta$-actin. Each experiment was repeated three times and the data are expressed as the mean \pm standard deviation $($ ( $\mathrm{P}<0.05$ or ${ }^{* *} \mathrm{P}<0.01$, compared with the shRNA-control group). sh, short hairpin.

Downregulation of biglycan inhibits the proliferation of colon cancer cells and causes cell cycle arrest. The effect of biglycan on the proliferation of colon cancer cells was assessed using a CCK-8 assay. As shown in Fig. 2A, a significant decrease in cell viability was observed between 2 and 3 days in the shRNA-biglycan-transfected HCT116 cells, compared with that in the shRNA-control-transfected or non-transfected cells $(\mathrm{P}<0.01)$. In addition, the effect of biglycan on the progression of the cell cycle was also detected by flow cytometry. The results revealed that the 
A

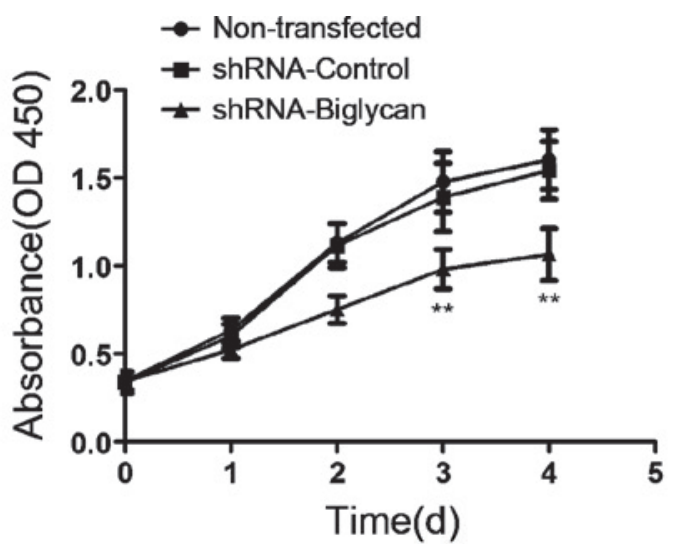

C

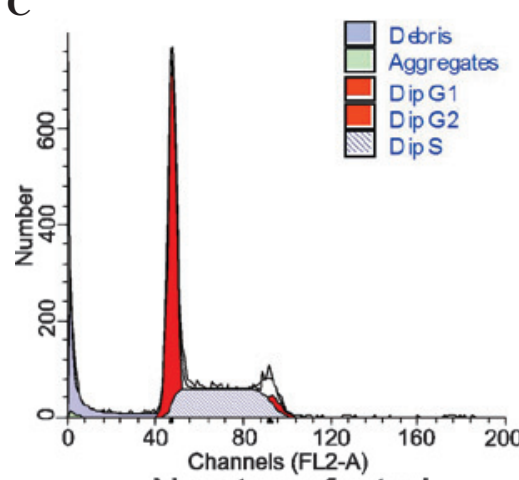

Non-transfected
B
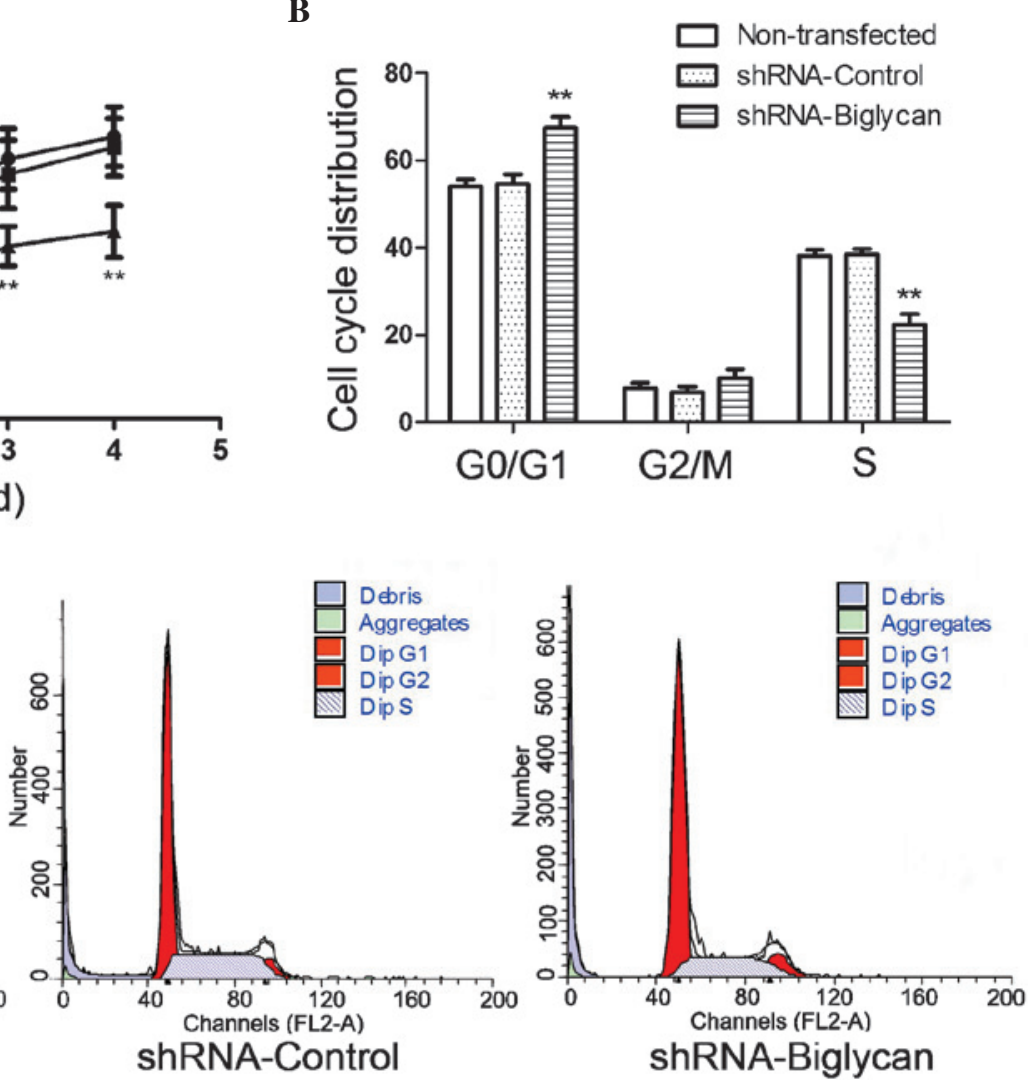

D

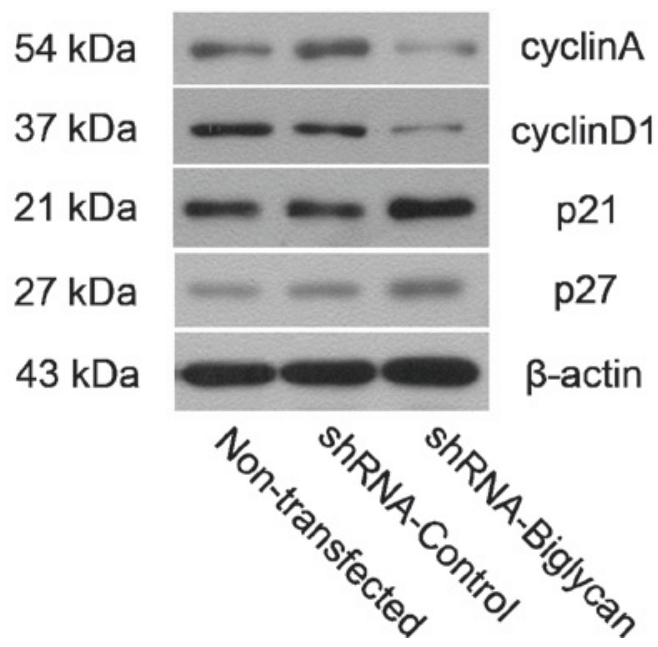

$\mathbf{E}$

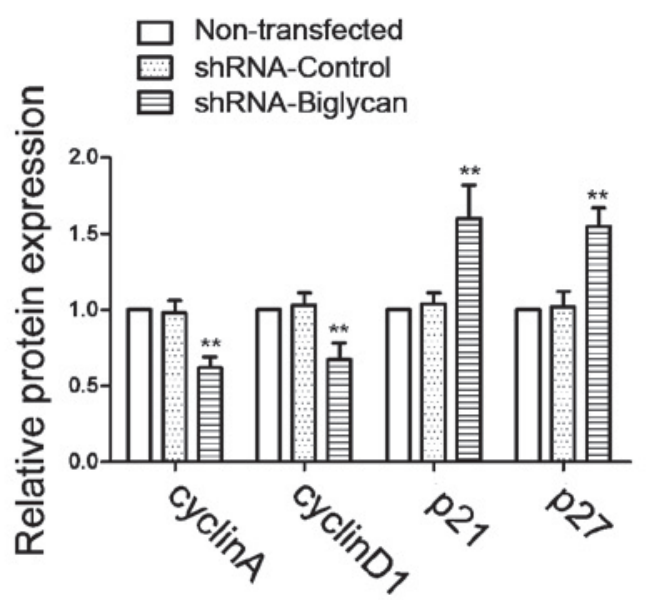

Figure 2. Downregulation of biglycan inhibits the proliferation of colon cancer cells and causes cell cycle arrest. (A) The effect of the downregulation of biglycan on HCT116 cell proliferation was measured using a cell counting kit-8 assay, with each group containing six technical replicates. (B and C) The effect of the downregulation of biglycan on the HCT116 cell cycle distribution was analyzed by flow cytometry. (D) The protein expression levels of cyclin A, cyclin D1, p21 and p27 were detected by western blotting, and the results obtained from a representative experiment are shown. (E) The relative protein expression of these proteins were normalized against $\beta$-actin. The data are expressed as the mean \pm standard deviation $\left({ }^{* *} \mathrm{P}<0.01\right.$, compared with the shRNA-control group). OD, optical density; sh, short hairpin.

downregulation of biglycan markedly affected the cell cycle distribution (Fig. 2B and C). The proportion of the cells in the G0/G1 phase was significantly increased in the shRNA-biglycan group compared with the shRNA-control or non-transfected groups $(\mathrm{P}<0.01)$, and the proportion of cells in $\mathrm{S}$ phase was decreased in the shRNA-biglycan group $(\mathrm{P}<0.01)$, which indicated that the cell cycle was arrested in the G0/G1 phase following biglycan silencing. To further confirm these results, the expression levels of the cell cycle markers, cyclin A and D1, and the cyclin-dependent kinase inhibitors, p21 and p27, were subsequently determined (14). The results from western blot analysis revealed that the downregulation of biglycan decreased the protein expression levels of cyclins A and D1 compared with the control cells, whereas the expression of p21 and p27 was increased following biglycan inhibition ( $\mathrm{P}<0.01$; Fig. $2 \mathrm{D}$ and $\mathrm{E})$. These 
A

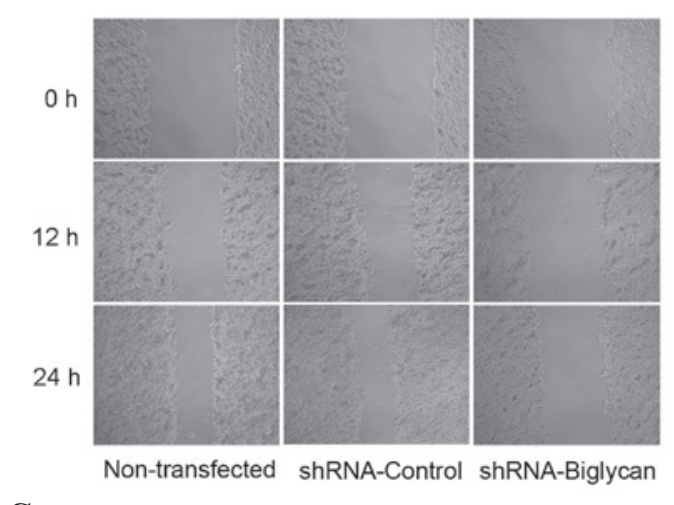

C

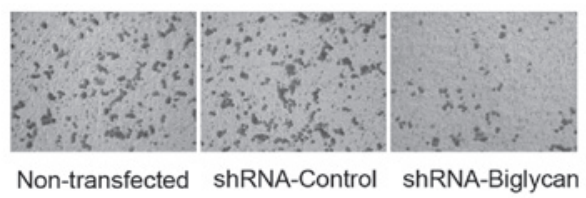

B

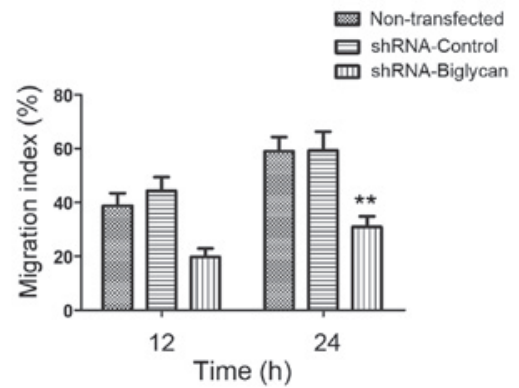

D

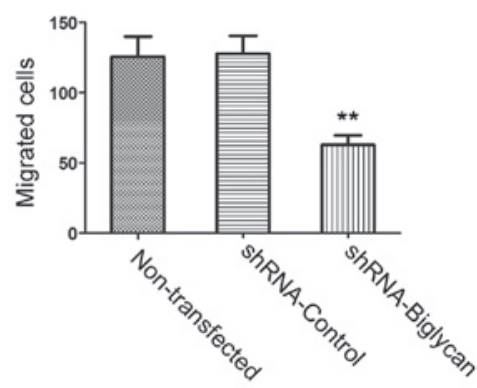

Figure 3. Downregulation of biglycan suppresses the migratory and invasive properties of colon cancer cells (magnification, x200). (A) The motility of shRNA-biglycan/control or non-transfected cells was determined using a scratch wound assay over a $24 \mathrm{~h}$ time period. (B) The migration rate was determined by the distance traveled by the cells to the front of the denuded area. (C) The invasion ability of cells in each group was measured using a Transwell assay. (D) The number of invasive cells were counted under an inverted microscope and cell numbers were plotted. The results obtained from a representative experiment are shown. The data are expressed as the mean \pm standard deviation $\left({ }^{* *} \mathrm{P}<0.01\right.$, compared with the shRNA-control group).. sh, short hairpin.

results suggested that the inhibition of biglycan expression arrested colon cancer cells in the G0/G1 phase, and inhibited cell proliferation.

Downregulation of biglycan suppresses the migratory and invasive properties of colon cancer cells. Previous studies demonstrated that biglycan exerts a distinct role in different tumor entities (15-17). To further investigate the underlying mechanism of biglycan in colon cancer, the effect of biglycan on colon cancer cell migration and invasion was determined. A scratch wound assay revealed that biglycan knockdown markedly reduced the spontaneous migration distance of shRNA-biglycan-transfected HCT116 cells compared with that of the shRNA-control or non-transfected cells at $24 \mathrm{~h}$ after the scratching of the cell surface $(\mathrm{P}<0.01$; Fig. $3 \mathrm{~A}$ and $\mathrm{B})$. Additionally, the effect of biglycan downregulation on the invasive ability of the cells was determined using a Transwell invasion assay. As shown in Fig. 3C and D, the number of invasive cells in the shRNA-biglycan group was significantly lower compared with that in the control group $(\mathrm{P}<0.01)$. These results indicated that the downregulation of biglycan expression inhibited the migration and invasive capability of the colon cancer cells.

Downregulation of biglycan activates the p38 signaling pathway and induces apoptosis in colon cancer cells. The effect of biglycan on the apoptosis of colon cancer cells was investigated by flow cytometry. As shown in Fig. 4A and B, the proportion of apoptotic cells in the shRNA-biglycan group $(18.45 \pm 1.09 \%)$ was significantly higher compared with that in the shRNA-control-transfected group $(3.51 \% \pm 0.16)$ or the non-transfected group $(4.24 \pm 0.61 \%)$, indicating that the downregulation of biglycan induced colon cancer cell apoptosis. Western blot analysis also revealed that the expression of the proapoptotic caspase-3 protein was markedly increased following the downregulation of biglycan (Fig. 4C and D), which was consistent with the flow cytometry results. Furthermore, a previous study demonstrated that the p38 signaling pathway has an important role in cell apoptosis (18). In the present study, we identified that the p38 signaling pathway was activated following biglycan knockdown; the addition of the p38 signal inhibitor, SB203580, effectively reversed the increased apoptotic cell number compared with the shRNA-biglycan group (7.56 $\pm 0.18 \%$; $\mathrm{P}<0.01$; Fig. 4A and B). These results indicated that biglycan may exert a role in regulating apoptosis in the colon cancer cells, specifically by inhibiting apoptosis via the regulation of the $\mathrm{p} 38$ MAPK signaling pathway.

\section{Discussion}

Previous studies demonstrated that biglycan exerts an important role in the mechanisms underlying the pathogenesis and progression of cancer, and abnormal expression levels of biglycan is usually indicative of a poor prognosis. For example, the expression levels of biglycan were significantly upregulated in esophageal squamous cell carcinoma (19), gastric (20), pancreatic (11) and colon (13) cancer; whereas decreased expression levels of biglycan were identified in human abdominal aortic aneurysms (21). This body of evidence indicates that biglycan may exert its distinct properties in different tumor entities. In the present study, the shRNA interference-mediated knockdown of biglycan markedly inhibited the proliferation of colon cancer cells, caused cell cycle arrest at the G0/G1 phase, suppressed the migratory 
A

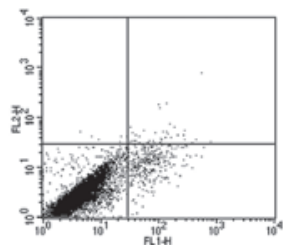

Non-transfected

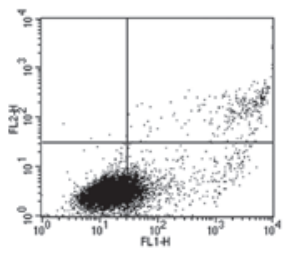

shRNA-Biglycan

C

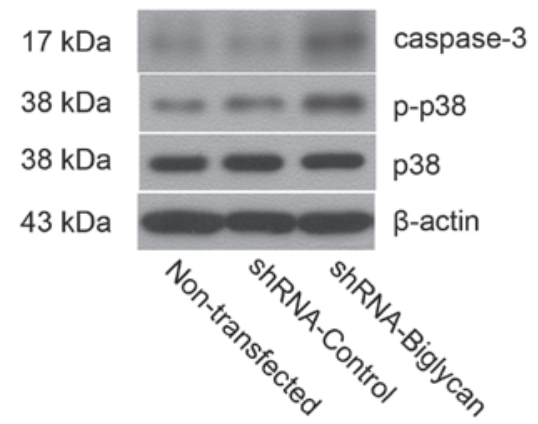

B

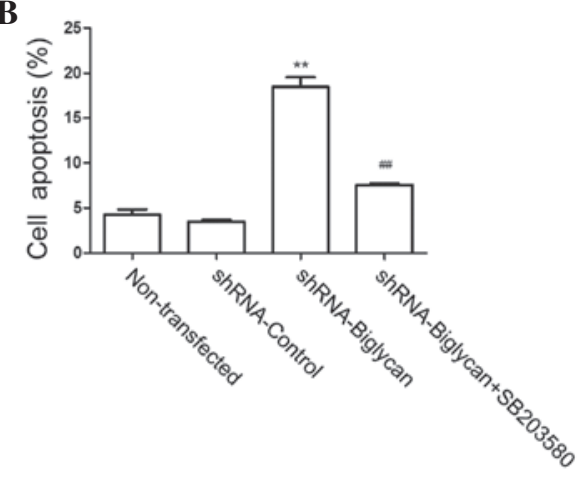

D

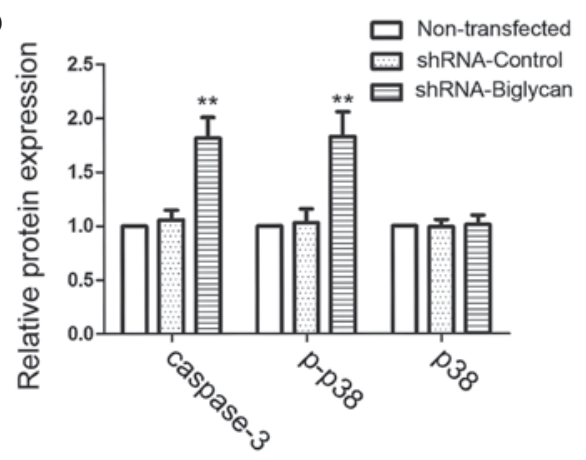

Figure 4. Downregulation of biglycan activates the p38 signaling pathway and induces apoptosis in colon cancer cells. (A) The effect of biglycan downregulation on the apoptosis of the HCT116 cells was detected using flow cytometry, and results are shown from a representative experiment. (B) The proportion of apoptotic cells in each group was determined. (C) The protein expression levels of caspase-3 and p-p38 were detected by western blotting, and the results obtained from a representative experiment are shown for each group. (D) The relative expression of these proteins was normalized against $\beta$-actin. The data are expressed as the mean \pm standard deviation $\left({ }^{* *} \mathrm{P}<0.01\right.$, compared with the shRNA-control group; ${ }^{\# \#} \mathrm{P}<0.01$, compared with the shRNA-biglycan group). p-, phosphorylated; sh, short hairpin.

and invasive properties of the HCT116 cells, and induced cell apoptosis. These results indicated that biglycan exerts an important role in the tumorigenesis and progression of colon cancer.

It is well established that the primary hallmarks of malignant tumors include uncontrolled tumor cell growth, dispersion and metastasis. The migration and invasion of the tumor cells is associated with the aggressive potential of cancer (22). Previous studies demonstrated that the extracellular matrix and the basement membrane were disrupted during tumorigenesis and metastasis $(23,24)$. Biglycan is an important component of the extracellular matrix. Mounting evidence supports the hypothesis that biglycan fulfils an oncogenic role in gastric cancer, and gastric cancer cells exhibited increased motility and invasive properties upon biglycan overexpression (17). In the present study, the shRNA-mediated knockdown of biglycan markedly reduced the spontaneous migration distance and the number of invasive colon cancer cells. Furthermore, it was observed that the shRNA-biglycan-transfected HCT116 cells exhibited a decreased rate of cell growth compared with that of the shRNA-control-transfected or non-transfected cells. The cell cycle progression analysis further revealed that the decreased expression of biglycan notably affected the cell cycle distribution. The proportion of cells in the G0/G1 phase was significantly increased, and the expression levels of the cell cycle-associated proteins, cyclins A and D1, were downregulated, whereas the levels of their inhibitory proteins, p21 and p27, were upregulated. These results were consistent with previously published results, which demonstrated that biglycan may promote cell proliferation and the migratory abilities of cells $(25,26)$. Furthermore, these results indirectly support our previous findings that biglycan overexpression promoted xenograft colon tumor growth, and that the upregulation of biglycan was associated with the malignancy in human colorectal cancer $(13,27)$. The results of the present study indicated that biglycan has an oncogenic role in the colon cancer process, which is associated with regulating the proliferation, migration and invasion of colon cancer cells.

Deregulated proliferation and inhibition of apoptosis promotes tumorigenesis. As reported previously, the p38 signaling pathway occupies a central role in the regulation of tumor cell proliferation and apoptosis (28). Multiple evidence supports the hypothesis that the p38 signaling pathway is activated in colon cancer cells and induces apoptosis. Furthermore, the activation of the p38 signaling pathway by biglycan has been demonstrated in multiple cell types $(29,30)$. To further investigate the mechanism of action of biglycan in regulating cell apoptosis, the effect of the p38 signal inhibitor, SB203580, was assessed in the present 
study. The results revealed that the knockdown of biglycan caused a significant increase in apoptotic cell numbers, in addition to the activation of the p38 signaling pathway. However, SB203580 effectively reversed the increase induced by biglycan silencing. These findings indicated that the inhibition of biglycan may induce cell apoptosis and that biglycan regulates the p38 signaling pathway by exerting an antiapoptotic effect in tumorigenesis.

In conclusion, the results in the present study demonstrated that decreased expression levels of biglycan inhibited cell proliferation, arrested the cell cycle at the G0/G1 phase, and suppressed the invasion and migration of colon cancer cells. Furthermore, the shRNA-mediated knockdown of biglycan induced apoptosis in colon cancer cells, mediated, at least in part, by an activation of the p38 signaling pathway. Therefore, biglycan may represent a target for gene therapy in the treatment of colon cancer.

\section{Acknowledgements}

The present study was supported by grants from the National Natural Science Foundation of China (no. 81201968), the Natural Science Foundation of Liaoning Province (no. 201102111) and the Doctoral Starting Foundation of Liaoning Province (no. 20091045).

\section{References}

1. Parkin DM, Bray F, Ferlay $\mathrm{J}$ and Pisani P: Global cancer statistics, 2002. CA Cancer J Clin 55: 74-108, 2005.

2. Cheng H,Zhang L, Cogdell DE, Zheng H, Schetter AJ, Nykter M, Harris CC, Chen K, Hamilton SR and Zhang W: Circulating plasma MiR-141 is a novel biomarker for metastatic colon cancer and predicts poor prognosis. PloS One 6: e17745, 2011.

3. Yang L, Parkin DM, Ferlay J, Li L and Chen Y: Estimates of cancer incidence in China for 2000 and projections for 2005 . Cancer Epidemiol Biomarkers Prev 14: 243-250, 2005.

4. Jemal A, Bray F, Center MM, Ferlay J, Ward E and Forman D: Global cancer statistics. CA Cancer J Clin 61: 69-90, 2011.

5. Wadhwa S, Embree MC, Bi Y and Young MF: Regulation, regulatory activities and function of biglycan. Crit Rev Eukaryot Gene Expr 14: 301-315, 2004.

6. Ameye L, Aria D, Jepsen K, Oldberg A, Xu T and Young MF: Abnormal collagen fibrils in tendons of biglycan/fibromodulin-deficient mice lead to gait impairment, ectopic ossification and osteoarthritis. FASEB J 16: 673-680, 2002.

7. Young MF, Bi Y, Ameye L and Chen XD: Biglycan knockout mice: New models for musculoskeletal diseases. Glycoconj J 19: 257-262, 2002

8. Nishino R, Honda M, Yamashita T, Takatori H, Minato H, Zen Y, Sasaki M, Takamura H, Horimoto K, Ohta T, et al: Identification of novel candidate tumour marker genes for intrahepatic cholangiocarcinoma. J Hepatol 49: 207-216, 2008.

9. Pan S, Cheng L, White JT, Lu W, Utleg AG, Yan X, Urban ND, Drescher CW, Hood L and Lin B: Quantitative proteomics analysis integrated with microarray data reveals that extracellular matrix proteins, catenins and $\mathrm{p} 53$ binding protein 1 are important for chemotherapy response in ovarian cancers. OMICS 13: 345-354, 2009.

10. Liu Y, Li W, Li X, Tai Y, Lü Q, Yang N and Jiang J: Expression and significance of biglycan in endometrial cancer. Arch Gynecol Obstet 289: 649-655, 2014.
11. Aprile G, Avellini C, Reni M, Mazzer M, Foltran L, Rossi D, Cereda S, Iaiza E, Fasola G and Piga A: Biglycan expression and clinical outcome in patients with pancreatic adenocarcinoma. Tumour Biol 34: 131-137, 2013.

12. Hu L, Duan YT, Li JF, Su LP, Yan M, Zhu ZG, Liu BY and Yang QM: Biglycan enhances gastric cancer invasion by activating FAK signaling pathway. Oncotarget 5: 1885-1896, 2014.

13. Gu X, Ma Y, Xiao J, Zheng H, Song C, Gong Y and Xing X: Up-regulated biglycan expression correlates with the malignancy in human colorectal cancers. Clin Exp Med 12: 195-199, 2012.

14. Weber CK, Sommer G, Michl P, Fensterer H, Weimer M, Gansauge F, Leder G, Adler G and Gress TM: Biglycan is overexpressed in pancreatic cancer and induces G1-arrest in pancreatic cancer cell lines. Gastroenterology 121: 657-667, 2001.

15. Recktenwald CV, Leisz S, Steven A, Mimura K, Müller A Wulfänger J, Kiessling R and Seliger B: HER-2/neu-mediated down-regulation of biglycan associated with altered growth properties. J Biol Chem 287: 24320-24329, 2012.

16. Makatsori E, Lamari FN, Theocharis AD, Anagnostides S, Hjerpe A, Tsegenidis T and Karamanos NK: Large matrix proteoglycans, versican and perlecan, are expressed and secreted by human leukemic monocytes. Anticancer Res 23: 3303-3309, 2003.

17. Hu L, Duan YT, Li JF, Su LP, Yan M, Zhu ZG, Liu BY and Yang QM: Biglycan enhances gastric cancer invasion by activating FAK signaling pathway. Oncotarget 5: 1885-1896, 2014.

18. Xia Z, Dickens M, Raingeaud J, Davis RJ and Greenberg ME: Opposing effects of ERK and JNK-p38 MAP kinases on apoptosis. Science 270: 1326-1331, 1995.

19. Zhu YH, Yang F, Zhang SS, Zeng TT, Xie X and Guan XY: High expression of biglycan is associated with poor prognosis in patients with esophageal squamous cell carcinoma. Int J Clin Exp Pathol 6: 2497-2505, 2013.

20. Wang B, Li GX, Zhang SG, Wang Q, Wen YG, Tang HM, Zhou CZ, Xing AY, Fan JW, Yan DW, et al: Biglycan expression correlates with aggressiveness and poor prognosis of gastric cancer. Exp Biol Med (Maywood) 236: 1247-1253, 2011.

21. Theocharis AD and Karamanos NK: Decreased biglycan expression and differential decorin localization in human abdominal aortic aneurysms. Atherosclerosis 165: 221-230, 2002.

22. Gupta GP and Massague J: Cancer metastasis: Building a framework. Cell 127: 679-695, 2006.

23. Lu P, Weaver VM and Werb Z: The extracellular matrix: A dynamic niche in cancer progression. J Cell Biol 196: 395-406, 2012.

24. Stetler-Stevenson WG, Aznavoorian S and Liotta LA: Tumor cell interactions with the extracellular matrix during invasion and metastasis. Annu Rev Cell Biol 9: 541-573, 1993.

25. Shimizu-Hirota R, Sasamura H, Kuroda M, Kobayashi E, Hayashi $M$ and Saruta T: Extracellular matrix glycoprotein biglycan enhances vascular smooth muscle cell proliferation and migration. Circ Res 94: 1067-1074, 2004.

26. Yamamoto K, Ohga N, Hida Y, Maishi N, Kawamoto T, Kitayama K, Akiyama K, Osawa T, Kondoh M, Matsuda K, et al: Biglycan is a specific marker and an autocrine angiogenic factor of tumour endothelial cells. Br J Cancer 106: 1214-1223, 2012.

27. Xing X, Gu X, Ma T and Ye H: Biglycan up-regulated vascular endothelial growth factor (VEGF) expression and promoted angiogenesis in colon cancer. Tumor Biol 36: 1773-1780, 2015.

28. Wagner EF and Nebreda AR: Signal integration by JNK and p38 MAPK pathways in cancer development. Nat Rev Cancer 9: 537-549, 2009.

29. Babelova A, Moreth K, Tsalastra-Greul W, Zeng-Brouwers J, Eickelberg O, Young MF, Bruckner P, Pfeilschifter J, Schaefer RM, Gröne HJ and Schaefer L: Biglycan, a danger signal that activates the NLRP3 inflammasome via toll-like and P2X receptors. J Biol Chem 284: 24035-24048, 2009.

30. Schaefer L, Babelova A, Kiss E, Hausser HJ, Baliova M, Krzyzankova M, Marsche G, Young MF, Mihalik D, Götte M, et al: The matrix component biglycan is proinflammatory and signals through Toll-like receptors 4 and 2 in macrophages. J Clin Invest 115: 2223-2233, 2005. 